\title{
The zero modes and zero resonances of massless Dirac operators
}

\author{
Yoshimi SAITŌ and Tomio UMEDA
}

(Received December 13, 2006)

\begin{abstract}
The zero modes and zero resonances of the Dirac operator $H=\alpha \cdot D+Q(x)$ are discussed, where $\alpha=\left(\alpha_{1}, \alpha_{2}, \alpha_{3}\right)$ is the triple of $4 \times 4$ Dirac matrices, $D=(1 / i) \nabla_{x}$, and $Q(x)=\left(q_{j k}(x)\right)$ is a $4 \times 4$ Hermitian matrix-valued function with $\left|q_{j k}(x)\right| \leq C\langle x\rangle^{-\rho}$, $\rho>1$. We shall show that every zero mode $f(x)$ is continuous on $\mathbb{R}^{3}$ and decays at infinity with the decay rate $|x|^{-2}$. Also, we shall show that $H$ has no zero resonance if $\rho>3 / 2$.

Key words: Dirac operators, Weyl-Dirac operators, zero modes, zero resonances, the limiting absorption principle.
\end{abstract}

\section{Introduction}

This paper is concerned with the massless Dirac operator

$$
H=\alpha \cdot D+Q(x), D=\frac{1}{i} \nabla_{x}, \quad x \in \mathbb{R}^{3},
$$

where $\alpha=\left(\alpha_{1}, \alpha_{2}, \alpha_{3}\right)$ is the triple of $4 \times 4$ Dirac matrices

$$
\alpha_{j}=\left(\begin{array}{cc}
\mathbf{0} & \sigma_{j} \\
\sigma_{j} & \mathbf{0}
\end{array}\right) \quad(j=1,2,3)
$$

with the $2 \times 2$ zero matrix $\mathbf{0}$ and the triple of $2 \times 2$ Pauli matrices

$$
\sigma_{1}=\left(\begin{array}{ll}
0 & 1 \\
1 & 0
\end{array}\right), \sigma_{2}=\left(\begin{array}{cc}
0 & -i \\
i & 0
\end{array}\right), \sigma_{3}=\left(\begin{array}{cc}
1 & 0 \\
0 & -1
\end{array}\right),
$$

and $Q(x)$ is a $4 \times 4$ Hermitian matrix-valued function decaying at infinity.

We would like to emphasize that one can regard the operator (1.1) as a generalization of the operator

$$
\alpha \cdot(D-A(x))+q(x) I_{4}
$$

where $(q, A)$ is an electromagnetic potential and $I_{4}$ is a $4 \times 4$ identity matrix, by taking $Q(x)$ to be $-\alpha \cdot A(x)+q(x) I_{4}$. In the case where $q(x) \equiv 0$, the 
operator (1.2) becomes of the form

$$
\alpha \cdot(D-A(x))=\left(\begin{array}{cc}
\mathbf{0} & \sigma \cdot(D-A(x)) \\
\sigma \cdot(D-A(x)) & \mathbf{0}
\end{array}\right) .
$$

The component $\sigma \cdot(D-A(x))$ is called the Weyl-Dirac operator. See Balinsky and Evans [6].

In the paper by Fröhlich, Lieb and Loss [16], it was found that the existence of zero modes (i.e., eigenfunctions with the zero eigenvalue) of the Weyl-Dirac operator plays a crucial role in the study of stability of Coulomb systems with magnetic fields. (For the precise definition of zero modes, see Definition 1.1 in the latter part of this section.) Loss and Yau [23] were the first to construct zero modes of the Weyl-Dirac operator and their results were usefully applied in [16]. Since then, the zero modes of the Dirac operator $\alpha \cdot(D-A(x))$, the Weyl-Dirac operator $\sigma \cdot(D-A(x))$ and the Pauli operator $\{\sigma \cdot(D-A(x))\}^{2}+q(x) I_{2}$ have attracted a considerable attention. It is now widely understood that the zero modes have deep and fruitful implications from the view point of mathematics as well as physics. See Adam, Muratori and Nash [1], [2], [3], Balinsky and Evans [5], [6], [7], Elton [11] and, Erdös and Solovej [12]. Also, see Bugliaro, Fefferman and Graf [9] and, Erdös and Solovej [13], [14], where their main concern is LiebThirring inequality for the Pauli operator with a strong magnetic fields and, as a by-product, an estimate of the density of zero modes of the Weyl-Dirac operators was obtained.

As for the two-dimensional case, Aharonov and Casher [4] are believed to be the first to construct examples of zero modes. See Erdös and Vougalter [15], Rozenblum and Shirokov [29] and Persson [24] for recent works.

We should like to note that the operator (1.1) also generalizes the Dirac operator of the form

$$
\alpha \cdot D+m(x) \beta+q(x) I_{4},
$$

where $m(x)$ is considered to be a variable mass, and $\beta$ is the $4 \times 4$ matrix defined by

$$
\beta=\left(\begin{array}{cc}
I_{2} & \mathbf{0} \\
\mathbf{0} & -I_{2}
\end{array}\right)
$$

Spectral properties of the operator (1.3) have been extensively studied in recent years. See Kalf and Yamada [19], Kalf, Okaji and Yamada [20], 
Schmidt and Yamada [30], Pladdy [25] and Yamada [35].

Finally, we would like to emphasize the significant role of the zero modes and zero resonances in the analysis of the asymptotic behavior, around the origin of the complex plane, of the resolvent of the operator $H$ given by (1.1). One can easily recognize the significance as is suggested by Jensen and Kato [18] on the Schrödinger operator.

Notation The upper and lower half planes $\mathbb{C}_{ \pm}$are defined by

$$
\mathbb{C}_{+}:=\{z \in \mathbb{C} \mid \operatorname{Im} z>0\}, \quad \mathbb{C}_{-}:=\{z \in \mathbb{C} \mid \operatorname{Im} z<0\}
$$

respectively. By $S\left(\mathbb{R}^{3}\right)$, we mean the Schwartz class of rapidly decreasing functions on $\mathbb{R}^{3}$, and we set $\mathcal{S}=\left[S\left(\mathbb{R}^{3}\right)\right]^{4}$.

By $L^{2}=L^{2}\left(\mathbb{R}^{3}\right)$, we mean the Hilbert space of square-integrable functions on $\mathbb{R}^{3}$, and we introduce a Hilbert space $\mathcal{L}^{2}$ by $\mathcal{L}^{2}=\left[L^{2}\left(\mathbb{R}^{3}\right)\right]^{4}$, where the inner product is given by

$$
(f, g)_{\mathcal{L}^{2}}=\sum_{j=1}^{4}\left(f_{j}, g_{j}\right)_{L^{2}}
$$

for $f={ }^{t}\left(f_{1}, f_{2}, f_{3}, f_{4}\right)$ and $g={ }^{t}\left(g_{1}, g_{2}, g_{3}, g_{4}\right)$.

By $L^{2, s}\left(\mathbb{R}^{3}\right)$, we mean the weighted $L^{2}$ space defined by

$$
L^{2, s}\left(\mathbb{R}^{3}\right):=\left\{u \mid\langle x\rangle^{s} u \in L^{2}\left(\mathbb{R}^{3}\right)\right\}
$$

with the inner product

$$
(u, v)_{L^{2, s}}:=\int_{\mathbb{R}^{3}}\langle x\rangle^{2 s} u(x) \overline{v(x)} d x,
$$

where

$$
\langle x\rangle=\sqrt{1+|x|^{2}} .
$$

We introduce the Hilbert space $\mathcal{L}^{2, s}=\left[L^{2, s}\left(\mathbb{R}^{3}\right)\right]^{4}$ with the inner product

$$
(f, g)_{\mathcal{L}^{2, s}}=\sum_{j=1}^{4}\left(f_{j}, g_{j}\right)_{L^{2, s} .}
$$

By $H^{\mu, s}\left(\mathbb{R}^{3}\right)$, we mean the weighted Sobolev space defined by

$$
H^{\mu, s}\left(\mathbb{R}^{3}\right):=\left\{u \in S^{\prime}\left(\mathbb{R}^{3}\right) \mid\langle x\rangle^{s}\langle D\rangle^{\mu} u \in L^{2}\left(\mathbb{R}^{3}\right)\right\}
$$


with the inner product

$$
(u, v)_{H^{\mu, s}}:=\left(\langle x\rangle^{s}\langle D\rangle^{\mu} u,\langle x\rangle^{s}\langle D\rangle^{\mu} v\right)_{L^{2}}
$$

where

$$
\langle D\rangle=\sqrt{1-\Delta} .
$$

In a similar fashion, we introduce the Hilbert space $\mathcal{H}^{\mu, s}=\left[H^{\mu, s}\left(\mathbb{R}^{3}\right)\right]^{4}$. Note that $H^{\mu, 0}\left(\mathbb{R}^{3}\right)$ coincides with the Sobolev space of order $\mu: H^{\mu}\left(\mathbb{R}^{3}\right)$, and by $\mathcal{H}^{\mu}$ we mean the Hilbert space $\left[H^{\mu}\left(\mathbb{R}^{3}\right)\right]^{4}$. Also note that $\mathcal{H}^{0,0}=\mathcal{L}^{2}$ and $\mathcal{H}^{0, s}=\mathcal{L}^{2, s}$

By $B(\mu, s ; \nu, t)$, we mean the set of all bounded linear operators from $H^{\mu, s}\left(\mathbb{R}^{3}\right)$ into $H^{\nu, t}\left(\mathbb{R}^{3}\right)$, and by $\mathcal{B}(\mu, s ; \nu, t)$, the set of all bounded linear operators from $\mathcal{H}^{\mu, s}$ into $\mathcal{H}^{\nu, t}$. For an operator $W \in B(\mu, s ; \nu, t)$, we define a copy of $W \in \mathcal{B}(\mu, s ; \nu, t)$ by

$$
\begin{aligned}
\mathcal{H}^{\mu, s} \ni f={ }^{t} & \left(f_{1}, f_{2}, f_{3}, f_{4}\right) \\
& \mapsto W f={ }^{t}\left(W f_{1}, W f_{2}, W f_{3}, W f_{4}\right) \in \mathcal{H}^{\nu, t} .
\end{aligned}
$$

Assumption (A) Each element $q_{j k}(x)(j, k=1, \ldots, 4)$ of $Q(x)$ is a measurable function satisfying

$$
\left|q_{j k}(x)\right| \leq C\langle x\rangle^{-\rho} \quad(\rho>1),
$$

where $C$ is a positive constant. Moreover, $Q(x)$ is a Hermitian matrix for each $x \in \mathbb{R}^{3}$.

Note that, under Assumption (A), the Dirac operator (1.1) is a selfadjoint operator in $\mathcal{L}^{2}$ with $\operatorname{Dom}(H)=\mathcal{H}^{1}$. The self-adjoint realization will be denoted by $H$ again. With an abuse of notation, we shall write $H f$ in the distributional sense for $f \in \mathcal{S}^{\prime}$ whenever it makes sense.

Definition 1.1 By a zero mode, we mean a function $f \in \operatorname{Dom}(H)$ which satisfies

$$
H f=0 .
$$

By a zero resonance, we mean a function $f \in \mathcal{L}^{2,-s} \backslash \mathcal{L}^{2}$, for some $s>0$, which satisfies $H f=0$ in the distributional sense.

It is evident that a zero mode of $H$ is an eigenfunction of $H$ corresponding to the eigenvalue 0, i.e., a zero mode is an element of $\operatorname{Ker}(H)$, the kernel 
of the self-adjoint operator $H$.

It would seem that there is no decisive definition of zero resonances. A common understanding of zero resonances in the literature is that a zero resonance is a non- $\mathcal{L}^{2}$ solution of $H f=0$ in a space slightly larger than $\mathcal{L}^{2}$. (See, for example, Jensen and Kato [18].) In dealing with zero resonances in Section 2 and later sections, we shall restrict ourselves to the case where $\rho>3 / 2$ and $0<s \leq \min \{3 / 2, \rho-1\}$.

Balinsky and Evans [6] is particularly interesting from our view point in the sense that they dealt with the Weyl-Dirac operator $\sigma \cdot(D-A(x))$ and showed that the set of magnetic fields which give rise to zero modes is rather "sparse."

In this paper, we investigate the zero modes and zero resonances of the operator $H$ in (1.1) under Assumption(A). Our goal is to establish a pointwise estimate of the zero modes as well as the continuity of the zero modes, and also to show that the zero resonances do not exist.

\section{Main results}

Theorem 2.1 Suppose Assumption (A) is satisfied. Let $f$ be a zero mode of the operator (1.1). Then

(i) the inequality

$$
|f(x)| \leq C\langle x\rangle^{-2}
$$

holds for all $x \in \mathbb{R}^{3}$, where the constant $C\left(=C_{f}\right)$ depends only on the zero mode $f$;

(ii) the zero mode $f$ is a continuous function on $\mathbb{R}^{3}$.

Remark 2.1 It is natural that zero modes exhibit only polynomial decays at infinity. In Loss and Yau [23], they considered the Weyl-Dirac operator $\sigma \cdot(D-A(x))$, and constructed two examples of pairs of a vector potential $A$ and a zero mode $\psi$. One of their examples shows that $A(x)=O\left(|x|^{-2}\right)$ and $\psi(x)=O\left(|x|^{-2}\right.$ ) at infinity. (Also, see examples in Adam, Muratori and Nash [1].) Thus, it is true that the decay rate in Theorem 2.1 is optimal at least for $\rho$ with $1<\rho \leq 2$.

Remark 2.2 In Bugliaro, Fefferman and Graf [9] and, Erdös and Solovej [13], [14], they established estimates of the density of zero modes of the Weyl-Dirac operator $\sigma \cdot(D-A(x))$. It is apparent that their estimates 
immediately imply estimates of each zero mode. These estimates of each zero mode are, however, quite unclear in terms of the decay rate at infinity because their estimates contain local lengthscales of the magnetic fields.

Theorem 2.2 below means that zero resonances do not exist under the restriction on $s$ mentioned after Definition 1.1. Accordingly, we need a larger $\rho$ than Theorem 2.1.

Theorem 2.2 Suppose Assumption (A) is satisfied with $\rho>3 / 2$. If $f$ belongs to $\mathcal{L}^{2,-s}$ for some $s$ with $0<s \leq \min \{3 / 2, \rho-1\}$ and satisfies $H f=0$ in the distributional sense, then $f \in \mathcal{H}^{1}$.

\section{A singular integral operator}

One of the ingredients of the proofs of the main theorems is a singular integral operator acting on four component vector functions. The singular integral operator we deal with in this section is defined by

$$
A f(x)=\int_{\mathbb{R}^{3}} i \frac{\alpha \cdot(x-y)}{4 \pi|x-y|^{3}} f(y) d y
$$

for

$$
f={ }^{t}\left(f_{1}, f_{2}, f_{3}, f_{4}\right) \in \mathcal{L}^{2},
$$

where $\alpha \cdot(x-y)$ means the sum of the matrix operation $\alpha_{j}$ for the four-vector $\left(x_{j}-y_{j}\right) f$ :

$$
\alpha \cdot(x-y) f=\sum_{j=1}^{3} \alpha_{j}\left(x_{j}-y_{j}\right) f .
$$

We shall need a few estimates of $A$ on $\mathcal{L}^{2}$ and on its subspaces.

Lemma 3.1 For each $f \in \mathcal{L}^{2}, A f(x)$ is defined for a.e. $x \in \mathbb{R}^{3}$. Moreover, $A$ is a bounded operator from $\mathcal{L}^{2}$ to $\mathcal{L}^{6}$, i.e., there exists a constant $C$ such that

$$
\|A f\|_{\mathcal{L}^{6}} \leq C\|f\|_{\mathcal{L}^{2}}
$$

for all $f \in \mathcal{L}^{2}$. 
Proof. Since $\alpha_{j}$ 's are unitary matrices and satisfy the anti-commutation relation $\alpha_{j} \alpha_{k}+\alpha_{k} \alpha_{j}=2 \delta_{j k} I_{4}$, we have

$$
|\alpha \cdot(x-y) f(y)|=|x-y||f(y)| .
$$

(Note that $|x-y|$ and $|f(y)|$ are the Euclidean norms of $\mathbb{R}^{3}$ and $\mathbb{R}^{4}$ respectively.) Therefore we get

$$
\begin{aligned}
|A f(x)| & \leq \frac{1}{4 \pi} \int_{\mathbb{R}^{3}} \frac{1}{|x-y|^{2}}|f(y)| d y \\
& =\frac{\pi}{2} I_{1}(|f|)(x),
\end{aligned}
$$

where $I_{1}$ is the Riesz potential; see Stein [31, p. 117]. We shall appeal two well-known facts (Stein $\left[31\right.$, p. 119]) that $I_{1}(u)(x)$ is finite for a.e. $x \in \mathbb{R}^{3}$ if $u \in L^{2}\left(\mathbb{R}^{3}\right)$, and that $I_{1}$ is a bounded operator from $L^{2}\left(\mathbb{R}^{3}\right)$ to $L^{6}\left(\mathbb{R}^{3}\right)$ (a special case of the Hardy-Littlewood-Sobolev inequality). These facts, together with (3.2), yield the conclusions of the lemma.

Lemma 3.2 Let $s \geq 1$. Then

$$
\|A f\|_{\mathcal{L}^{2}} \leq C\|f\|_{\mathcal{L}^{2, s}}
$$

for all $f \in \mathcal{L}^{2, s}$.

Proof. In view of (3.2), it is sufficient to show that the Riesz potential $I_{1}$ is a bounded operator from $L^{2, s}\left(\mathbb{R}^{3}\right)$ to $L^{2}\left(\mathbb{R}^{3}\right)$.

Let $u \in S\left(\mathbb{R}^{3}\right)$. Then we have

$$
I_{1}(u)=\overline{\mathcal{F}}\left[\frac{1}{2 \pi|\xi|}\right] \mathcal{F} u,
$$

where $\mathcal{F}$ and $\overline{\mathcal{F}}$ denote the Fourier transform and the inverse Fourier transform respectively (see [31, p. 117]), and

$$
\mathcal{F} u(\xi)=\hat{u}(\xi)=\frac{1}{(2 \pi)^{3 / 2}} \int_{\mathbb{R}^{3}} e^{-i x \cdot \xi} u(x) d x .
$$

It follows from (3.3) and the Plancherel theorem that

$$
\left\|I_{1}(u)\right\|_{L^{2}}^{2}=\frac{1}{4 \pi^{2}} \int_{\mathbb{R}^{3}} \frac{1}{|\xi|^{2}}|\hat{u}(\xi)|^{2} d \xi .
$$

If we apply the Hardy inequality (which is referred to as the uncertainty principle lemma in $[28$, p. 169$]$; also see $[21$, p. 4.50$]$ ) to the right hand side 
of (3.4), we get

$$
\begin{aligned}
\frac{1}{4 \pi^{2}} \int_{\mathbb{R}^{3}} \frac{1}{|\xi|^{2}}|\hat{u}(\xi)|^{2} d \xi & \leq \frac{1}{\pi^{2}} \int_{\mathbb{R}^{3}}\left|\nabla_{\xi} \hat{u}(\xi)\right|^{2} d \xi \\
& =\frac{1}{\pi^{2}} \int_{\mathbb{R}^{3}}|x u(x)|^{2} d x .
\end{aligned}
$$

Combining (3.4) and (3.5), we obtain

$$
\left\|I_{1}(u)\right\|_{L^{2}} \leq \frac{1}{\pi}\|x u\|_{L^{2}} \leq \frac{1}{\pi}\|u\|_{L^{2, s}}
$$

for $u \in S\left(\mathbb{R}^{3}\right)$, where we have used the hypothesis $s \geq 1$. Since $S\left(\mathbb{R}^{3}\right)$ is dense in $L^{2, s}\left(\mathbb{R}^{3}\right)$, it follows from (3.6) that $I_{1}$ is bounded from $L^{2, s}\left(\mathbb{R}^{3}\right)$ to $L^{2}\left(\mathbb{R}^{3}\right)$.

We introduce a class of functions which is necessary to establish an $\mathcal{L}^{\infty}$ estimate of the operator $A$. For $q \geq 1$, we define

$$
L_{u l}^{q}\left(\mathbb{R}^{3}\right)=\left\{u \in L_{\mathrm{loc}}^{q}\left(\mathbb{R}^{3}\right) \mid\|u\|_{L_{u l}^{q}}:=\sup _{x \in \mathbb{R}^{3}}\|u\|_{L^{q}(B(x ; 1))}<\infty\right\},
$$

where $B(x ; 1)=\left\{y \in \mathbb{R}^{3}|| x-y \mid \leq 1\right\}$, and define

$$
\mathcal{L}_{u l}^{q}=\left[L_{u l}^{q}\left(\mathbb{R}^{3}\right)\right]^{4}, \quad\|f\|_{\mathcal{L}_{u l}^{q}}=\sum_{k=1}^{4}\left\|f_{k}\right\|_{L_{u l}^{q} .} .
$$

Lemma 3.3 Let $1<p<3<q<+\infty$. Then there exists a constant $C_{p q}$ such that

$$
\|A f\|_{\mathcal{L}^{\infty}} \leq C_{p q}\left(\|f\|_{\mathcal{L}^{p}}+\|f\|_{\mathcal{L}_{u l}^{q}}\right)
$$

for all $f \in \mathcal{L}^{p} \cap \mathcal{L}_{u l}^{q}$. In particular,

$$
\|A f\|_{\mathcal{L}^{\infty}} \leq C_{p q}\left(\|f\|_{\mathcal{L}^{p}}+\|f\|_{\mathcal{L}^{q}}\right)
$$

for all $f \in \mathcal{L}^{p} \cap \mathcal{L}^{q}$.

Proof. By virtue of (3.2), we only have to prove that there exists a constant $C_{p q}^{\prime}$ such that

$$
\left\|I_{1}(u)\right\|_{L^{\infty}} \leq C_{p q}^{\prime}\left(\|u\|_{L^{p}}+\|u\|_{L_{u l}^{q}}\right) \quad \text { for } u \in L^{p}\left(\mathbb{R}^{3}\right) \cap L_{u l}^{q}\left(\mathbb{R}^{3}\right) .
$$

Since each $u \in L^{p}\left(\mathbb{R}^{3}\right) \cap L_{u l}^{q}\left(\mathbb{R}^{3}\right)$ can be decomposed as

$$
u=v_{+}-v_{-}+i\left(w_{+}-w_{-}\right),
$$




$$
v_{ \pm} \geq 0, w_{ \pm} \geq 0, v_{ \pm}, w_{ \pm} \in L^{p}\left(\mathbb{R}^{3}\right) \cap L_{u l}^{q}\left(\mathbb{R}^{3}\right)
$$

we shall prove (3.7) for $u \geq 0$.

Let $u \in L^{p}\left(\mathbb{R}^{3}\right) \cap L_{u l}^{q}\left(\mathbb{R}^{3}\right)$ be given, and let satisfy $u \geq 0$. Then one can find a sequence $\left\{\varphi_{n}\right\} \subset C_{0}^{\infty}\left(\mathbb{R}^{3}\right)$ such that

$$
0 \leq \varphi_{n} \leq u, \quad \varphi_{n} \rightarrow u \quad \text { in } L^{p}\left(\mathbb{R}^{3}\right) .
$$

(First cut $u$ as $\chi_{B(0 ; n)}(x) u$ by multiplying a characteristic function $\chi_{B(0 ; n)}$ of the ball $B(0 ; n)$ with center at the origin and radius $n$, then use the mollifier.) For each $n$, we decompose as

$$
\begin{aligned}
I_{1}\left(\varphi_{n}\right)(x) & =\int_{\mathbb{R}^{3}} \frac{1}{2 \pi^{2}|x-y|^{2}} \varphi_{n}(y) d y \\
& =h_{0} * \varphi_{n}(x)+h_{1} * \varphi_{n}(x),
\end{aligned}
$$

where

$$
\begin{aligned}
& h_{0}(x)=\chi_{B(0 ; 1)}(x) \frac{1}{2 \pi^{2}|x|^{2}}, \\
& h_{1}(x)=\left(1-\chi_{B(0 ; 1)}(x)\right) \frac{1}{2 \pi^{2}|x|^{2}} .
\end{aligned}
$$

(One should note that the integral on the right hand side of (3.9) converges because of the fact that $\varphi_{n} \in C_{0}^{\infty}\left(\mathbb{R}^{3}\right)$.) If we apply the Hölder inequality to $h_{0} * \varphi_{n}$, then we get

$$
\begin{aligned}
\left|h_{0} * \varphi_{n}(x)\right| & \leq \frac{1}{2 \pi^{2}}\left\{\int_{|x-y| \leq 1} \frac{1}{|x-y|^{2 q^{\prime}}} d y\right\}^{1 / q^{\prime}}\left\|\varphi_{n}\right\|_{L^{q}(B(x ; 1))} \\
& \leq C_{q}^{\prime}\|u\|_{L^{q}(B(x ; 1))} \quad\left(\frac{1}{q^{\prime}}=1-\frac{1}{q}\right)
\end{aligned}
$$

where we have used the fact that $2 q^{\prime}<3(\because q>3$ by assumption $)$ and (3.8). Similarly, if we apply the Hölder inequality to $h_{1} * \varphi_{n}$, we obtain

$$
\begin{aligned}
\left|h_{1} * \varphi_{n}(x)\right| & \leq \frac{1}{2 \pi^{2}}\left\{\int_{|x-y| \geq 1} \frac{1}{|x-y|^{2 p^{\prime}}} d y\right\}^{1 / p^{\prime}}\left\|\varphi_{n}\right\|_{L^{p}(\mathbb{R})} \\
& \leq C_{p}^{\prime}\|u\|_{L^{p}\left(\mathbb{R}^{3}\right)} \quad\left(\frac{1}{p^{\prime}}=1-\frac{1}{p}\right)
\end{aligned}
$$

where we have used the fact that $2 p^{\prime}>3(\because 1<p<3$ by assumption $)$ and 
(3.8). If follows from (3.9), (3.10) and (3.11) that

$$
\begin{aligned}
\left|I_{1}\left(\varphi_{n}\right)(x)\right| & \leq C_{p q}^{\prime}\left(\|u\|_{L^{q}(B(x ; 1))}+\|u\|_{L^{p}\left(\mathbb{R}^{3}\right)}\right) \\
& \leq C_{p q}^{\prime}\left(\|u\|_{L_{u l}^{q}}+\|u\|_{L^{p}}\right) .
\end{aligned}
$$

Recall that the Riesz potential $I_{1}$ is a bounded operator from $L^{p}\left(\mathbb{R}^{3}\right)$ to $L^{r}\left(\mathbb{R}^{3}\right),\left(r^{-1}=p^{-1}-3^{-1}\right)$, because of the Hardy-Littlewood-Sobolev inequality. This fact, together with (3.8), implies that there exists a subsequence $\left\{\varphi_{n^{\prime}}\right\}$ such that $I_{1}\left(\varphi_{n^{\prime}}\right)(x) \rightarrow I_{1}(u)(x)$ for a.e. $x \in \mathbb{R}^{3}$. Thus taking the limit of (3.12), along with the subsequence, gives (3.7).

\section{Estimates of the resolvents}

Another ingredient of the proofs of the main theorems is the limiting absorption principle (LAP) for the free Dirac operator

$$
H_{0}=\alpha \cdot D .
$$

We note that $H_{0}$ with $\operatorname{Dom}\left(H_{0}\right)=\mathcal{H}^{1}$ is a self-adjoint operator in $\mathcal{L}^{2}$. The self-adjoint realization will be denoted by $H_{0}$ again. It is well-known that the spectrum $\sigma\left(H_{0}\right)$ coincides with the whole real line $\mathbb{R}$. With an abuse of notation again, we shall write $H_{0} f$ for $f \in \mathcal{S}^{\prime}$.

We first prepare a lemma, which will be needed in the proof of Theorem 2.2 in Section 5.

Lemma 4.1 If $f \in \mathcal{L}^{2}$ and $(\alpha \cdot D) f \in \mathcal{L}^{2}$, then $f \in \mathcal{H}^{1}$.

Proof. We take the Fourier transform of $(\alpha \cdot D) f$, and we have

$$
\mathcal{F}[(\alpha \cdot D) f]=(\alpha \cdot \xi) \hat{f} .
$$

Then by using assumption of the lemma and (4.2), we see that

$$
\begin{aligned}
+\infty>\|(\alpha \cdot D) f\|_{\mathcal{L}^{2}}^{2} & =\int_{\mathbb{R}^{3}}|(\alpha \cdot \xi) \hat{f}(\xi)|^{2} d \xi \\
& =\int_{\mathbb{R}^{3}}\langle(\alpha \cdot \xi) \hat{f}(\xi),(\alpha \cdot \xi) \hat{f}(\xi)\rangle_{\mathbb{C}} d \xi \\
& =\int_{\mathbb{R}^{3}}|\xi|^{2}|\hat{f}(\xi)|^{2} d \xi,
\end{aligned}
$$

where $\langle\cdot, \cdot\rangle_{\mathbb{C}}$ denotes the inner product of $\mathbb{C}^{4}$. In the third equality of (4.3), we have used the anti-commutation relation. Since, by assumption of the 
lemma, $f \in \mathcal{L}^{2}$, the conclusion of the lemma follows from (4.3).

The task in the rest of this section is to prove the following theorem, which is essential in the proofs of the main theorems in Section 5.

Theorem 4.1 If $f \in \mathcal{L}^{2,-3 / 2}$ and $H_{0} f \in \mathcal{L}^{2, s}$ for some $s>1 / 2$, then $A H_{0} f=f$.

As was indicated at the beginning of this section, the ingredient of the proof of Theorem 4.1 is the LAP for the free Dirac operator $H_{0}$. Our idea of proving it is based on a decomposition of the resolvent

$$
R_{0}(z)=\left(H_{0}+z\right) \Gamma_{0}\left(z^{2}\right) \text { on } \mathcal{L}^{2}, \quad \operatorname{Im} z \neq 0,
$$

where

$$
R_{0}(z)=\left(H_{0}-z\right)^{-1}
$$

and $\Gamma_{0}(z)$ in (4.4) denotes the copy of the resolvent $\Gamma_{0}(z)=(-\Delta-z)^{-1}$ of the negative Laplacian

$$
-\Delta=-\left(\frac{\partial^{2}}{\partial x_{1}^{2}}+\frac{\partial^{2}}{\partial x_{2}^{2}}+\frac{\partial^{2}}{\partial x_{3}^{2}}\right) .
$$

See (1.5) for the definition of the copy of an operator. In other words, we shall not distinguish between $\Gamma_{0}(z)$ in $L^{2}\left(\mathbb{R}^{3}\right)$ and $\Gamma_{0}(z)$ in $\mathcal{L}^{2}$. We believe this will not cause any confusion.

A formal computation, based on the anti-commutation relation, shows that

$$
H_{0}^{2}=-\Delta I_{4},
$$

from which one can deduce (4.4). The decomposition (4.4) was first exploited in Balslev and Hellfer [8]. Similar decomposition was also adopted in Pladdy, Saitō and Umeda [26], [27].

We shall divide the rest of this section into two subsections, because the proof of Theorem 4.1 is lengthy.

\subsection{The resolvent of the negative Laplacian}

In this subsection, we shall state several lemmas, which are actually well-known and reproductions of results in Jensen and Kato [18] and Kuroda [21], [22]. We shall do this for our later purpose as well as for the reader's convenience. 
We first recall that the resolvent of $-\Delta$ can be represented as an integral operator:

$$
\Gamma_{0}(z) u(x)=\int_{\mathbb{R}^{3}} \frac{e^{i \sqrt{z}|x-y|}}{4 \pi|x-y|} u(y) d y, \quad u \in L^{2}\left(\mathbb{R}^{3}\right)
$$

for $z \in \mathbb{C} \backslash[0,+\infty)$, where $\operatorname{Im} \sqrt{z}>0$.

We next recall well-known inequalities (e.g., [10, Appendix A], [22, p. 162], [34, Lemma 11.1]), which will be repeatedly used in the present paper:

$$
\int_{\mathbb{R}^{3}} \frac{1}{|x-y|^{2}\langle y\rangle^{\gamma}} d y \leq C_{\gamma} \begin{cases}\langle x\rangle^{-\gamma+1} & \text { if } 1<\gamma<3, \\ \langle x\rangle^{-2} \log (1+\langle x\rangle) & \text { if } \gamma=3, \\ \langle x\rangle^{-2} & \text { if } \gamma>3 .\end{cases}
$$

Lemma 4.2 Let $s, s^{\prime}>1 / 2$ and $s+s^{\prime}>2$. Then

$$
\iint_{\mathbb{R}^{3} \times \mathbb{R}^{3}}\langle x\rangle^{-2 s^{\prime}} \frac{1}{|x-y|^{2}}\langle y\rangle^{-2 s} d x d y<+\infty .
$$

Proof. We may assume, with no loss of generality, that $s<3$. Then, by an inequality in (4.8), we have

$$
\int_{\mathbb{R}^{3}} \frac{1}{|x-y|^{2}}\langle y\rangle^{-2 s} d y \leq C_{s}\langle x\rangle^{-2 s+1} .
$$

Since $-2 s^{\prime}-2 s+1<-3$ by assumption of the lemma, we see that (4.10) implies (4.9).

It follows from (4.7) and Lemma 4.2 that the operator

$$
K(z):=\langle x\rangle^{-s^{\prime}} \Gamma_{0}(z)\langle x\rangle^{-s},
$$

which is represented as

$$
K(z) u(x)=\int_{\mathbb{R}^{3}}\langle x\rangle^{-s^{\prime}} \frac{e^{i \sqrt{z}|x-y|}}{4 \pi|x-y|}\langle y\rangle^{-s} u(y) d y,
$$

belongs to the Hilbert-Schmidt class on $L^{2}\left(\mathbb{R}^{3}\right)$ for $z \in \mathbb{C} \backslash[0,+\infty)$ :

$$
\|K(z)\|_{\mathrm{HS}}^{2}=\iint_{\mathbb{R}^{3} \times \mathbb{R}^{3}}\langle x\rangle^{-2 s^{\prime}}\left|\frac{e^{i \sqrt{z}|x-y|}}{4 \pi|x-y|}\right|^{2}\langle y\rangle^{-2 s} d x d y<+\infty,
$$


where $\|\cdot\|_{\text {HS }}$ denotes the Hilbert-Schmidt norm. Note that

$$
\begin{aligned}
& \left\|K\left(z_{1}\right)-K\left(z_{2}\right)\right\|_{\mathrm{HS}}^{2} \\
& =\iint_{\mathbb{R}^{3} \times \mathbb{R}^{3}}\langle x\rangle^{-2 s^{\prime}}\left|\frac{e^{i \sqrt{z_{1}}|x-y|}}{4 \pi|x-y|}-\frac{e^{i \sqrt{z_{2}}|x-y|}}{4 \pi|x-y|}\right|^{2}\langle y\rangle^{-2 s} d x d y
\end{aligned}
$$

for all $z_{1}, z_{2} \in \mathbb{C} \backslash[0,+\infty)$. It follows from (4.12) that $K(z)$ is continuous, with respect to the Hilbert-Schmidt norm topology, on $\mathbb{C} \backslash[0,+\infty)$. Furthermore, we can deduce from (4.9), (4.12) and Lebesgue's convergence theorem that $K(z)$ can be continuously extended, with respect to the HilbertSchmidt norm topology, as follows:

$$
\widetilde{K}(z)= \begin{cases}K(z) & \text { if } z \in \mathbb{C} \backslash[0,+\infty) \\ K^{+}(\lambda) & \text { if } z=\lambda+i 0, \lambda \geq 0 \\ K^{-}(\lambda) & \text { if } z=\lambda-i 0, \quad \lambda \geq 0\end{cases}
$$

where $K^{+}(\lambda)$ and $K^{-}(\lambda)$ for $\lambda>0$ are defined by

$$
K^{ \pm}(\lambda) u(x)=\int_{\mathbb{R}^{3}}\langle x\rangle^{-s^{\prime}} \frac{e^{ \pm i \sqrt{\lambda}|x-y|}}{4 \pi|x-y|}\langle y\rangle^{-s} u(y) d y,
$$

and

$$
K^{+}(0) u(x)=K^{-}(0) u(x)=\int_{\mathbb{R}^{3}}\langle x\rangle^{-s^{\prime}} \frac{1}{4 \pi|x-y|}\langle y\rangle^{-s} u(y) d y .
$$

For a later purpose, it is convenient to introduce a subset of the Riemann surface of $\sqrt{z}$ as follows:

$$
\begin{aligned}
\Pi_{(0,+\infty)} & \\
:=(\mathbb{C} \backslash(0,+\infty)) & \cup\{z=\lambda+i 0 \mid \lambda>0\} \\
& \cup\{z=\lambda-i 0 \mid \lambda>0\} .
\end{aligned}
$$

Thus, we can say that $\widetilde{K}(z)$ defined by $(4.13)-(4.15)$ is continuous on $\Pi_{(0,+\infty)}$ with respect to the Hilbert-Schmidt norm topology.

In view of (4.11), we see that $\Gamma_{0}(z), z \in \mathbb{C} \backslash[0,+\infty)$, is a HilbertSchmidt operator from $L^{2, s}\left(\mathbb{R}^{3}\right)$ to $L^{2,-s^{\prime}}\left(\mathbb{R}^{3}\right)$. Hence, in particular, $\Gamma_{0}(z) \in$ $B\left(0, s ; 0,-s^{\prime}\right)$, and

$$
\left\|\Gamma_{0}(z)\right\|_{B\left(0, s ; 0,-s^{\prime}\right)} \leq\|K(z)\|_{\mathrm{HS}} .
$$


Since we have the inequality

$$
\begin{aligned}
\left\|\Gamma_{0}\left(z_{1}\right)-\Gamma_{0}\left(z_{2}\right)\right\|_{B\left(0, s ; 0,-s^{\prime}\right)} \leq\left\|K\left(z_{1}\right)-K\left(z_{2}\right)\right\|_{\mathrm{HS}} \\
\quad\left(z_{1}, z_{2} \in \mathbb{C} \backslash[0,+\infty)\right),
\end{aligned}
$$

we conclude from (4.13) and (4.17) that $\Gamma_{0}(z) \in B\left(0, s ; 0,-s^{\prime}\right)$ can be continuously extended as follows:

$$
\widetilde{\Gamma}_{0}(z)= \begin{cases}\Gamma_{0}(z) & \text { if } z \in \mathbb{C} \backslash[0,+\infty), \\ \Gamma_{0}^{+}(\lambda) & \text { if } z=\lambda+i 0, \lambda \geq 0, \\ \Gamma_{0}^{-}(\lambda) & \text { if } z=\lambda-i 0, \lambda \geq 0,\end{cases}
$$

where

$$
\Gamma_{0}^{ \pm}(\lambda):=\langle x\rangle^{s^{\prime}} K^{ \pm}(\lambda)\langle x\rangle^{s}=\lim _{\epsilon \downarrow 0} \Gamma_{0}(\lambda \pm i \epsilon) \quad \text { in } B\left(0, s ; 0,-s^{\prime}\right) .
$$

We must remark that

$$
\Gamma_{0}^{+}(0) u(x)=\Gamma_{0}^{-}(0) u(x)=\int_{\mathbb{R}^{3}} \frac{1}{4 \pi|x-y|} u(y) d y,
$$

and that

$$
\Gamma_{0}^{ \pm}(\lambda) u(x)=\int_{\mathbb{R}^{3}} \frac{e^{ \pm i \sqrt{\lambda}|x-y|}}{4 \pi|x-y|} u(y) d y .
$$

Thus

$$
\widetilde{\Gamma}_{0}(\lambda+i 0) \neq \widetilde{\Gamma}_{0}(\lambda-i 0), \quad \lambda>0 .
$$

Note that the equality (4.19) allows us to use the notation

$$
\widetilde{\Gamma}_{0}(0)\left(=\Gamma_{0}^{+}(0)=\Gamma_{0}^{-}(0)\right) .
$$

With the notation introduced in (4.16), we can say that $\widetilde{\Gamma}_{0}(z)$ is a $B\left(0, s ; 0,-s^{\prime}\right)$-valued continuous function on $\Pi_{(0,+\infty)}$.

The following Lemmas 4.3 and 4.4 are variants of Lemma 2.1 of Jensen and Kato [18], although we shall give their proofs.

Lemma 4.3 Let $s$, $s^{\prime}$ satisfy the same assumption as in Lemma 4.2, and let $\mu \in \mathbb{R}$. Then $\widetilde{\Gamma}_{0}(z)$ is a $B\left(\mu, s ; \mu,-s^{\prime}\right)$-valued continuous function on $\Pi_{(0,+\infty)}$. 
Proof. As was mentioned before, $\widetilde{\Gamma}_{0}(z)$ defined by $(4.18)$ is a $B\left(0, s ; 0,-s^{\prime}\right)$ valued continuous function on $\Pi_{(0,+\infty)}$. Then the lemma directly follows from the inequalities

$$
\left\|\Gamma_{0}(z)\right\|_{B\left(\mu, s ; \mu,-s^{\prime}\right)} \leq\left\|\Gamma_{0}(z)\right\|_{B\left(0, s ; 0,-s^{\prime}\right)}, \quad z \in \mathbb{C} \backslash[0,+\infty)
$$

and

$$
\begin{array}{r}
\left\|\Gamma_{0}\left(z_{1}\right)-\Gamma_{0}\left(z_{2}\right)\right\|_{B\left(\mu, s ; \mu,-s^{\prime}\right)} \leq\left\|\Gamma_{0}\left(z_{1}\right)-\Gamma_{0}\left(z_{2}\right)\right\|_{B\left(0, s ; 0,-s^{\prime}\right)} \\
\left(z_{1}, z_{2} \in \mathbb{C} \backslash[0,+\infty) .\right.
\end{array}
$$

In order to show (4.21), we shall use the fact that

$$
\langle D\rangle^{\mu} \Gamma_{0}(z) u=\Gamma_{0}(z)\langle D\rangle^{\mu} u
$$

for $u \in S\left(\mathbb{R}^{3}\right)$ and $z \in \mathbb{C} \backslash[0,+\infty)$. We then have

$$
\begin{aligned}
\left\|\Gamma_{0}(z) u\right\|_{H^{\mu,-s}} & =\left\|\langle D\rangle^{\mu} \Gamma_{0}(z) u\right\|_{L^{2,-s^{\prime}}}=\left\|\Gamma_{0}(z)\langle D\rangle^{\mu} u\right\|_{L^{2,-s^{\prime}}} \\
& \leq\left\|\Gamma_{0}(z)\right\|_{B\left(0, s ; 0,-s^{\prime}\right)}\left\|\langle D\rangle^{\mu} u\right\|_{L^{2, s}} \\
& =\left\|\Gamma_{0}(z)\right\|_{B\left(0, s ; 0,-s^{\prime}\right)}\|u\|_{H^{\mu, s}}
\end{aligned}
$$

which implies (4.21). In a similar fashion, we can prove (4.22).

Remark 4.1 We should remark that $H^{\mu, s}\left(\mathbb{R}^{3}\right)$ in Lemma 4.3 is a subset of $L^{2}\left(\mathbb{R}^{3}\right)$ for $\mu \geq 0$, but not necessarily for $\mu<0$. Thus, the domain of $\widetilde{\Gamma}_{0}(z)$ depends on $\mu$ and $s$. Nonetheless, we have the unique representation of $\widetilde{\Gamma}_{0}(z)$ on $S\left(\mathbb{R}^{3}\right)$, a dense subset of $H^{\mu, s}\left(\mathbb{R}^{3}\right)$ :

$$
\widetilde{\Gamma}_{0}(z) u(x)=\int_{\mathbb{R}^{3}} \frac{e^{i \sqrt{z}|x-y|}}{4 \pi|x-y|} u(y) d y, \quad u \in S\left(\mathbb{R}^{3}\right),
$$

for every $z \in \Pi_{(0,+\infty)}$, where $\operatorname{Im} \sqrt{z} \geq 0$. This representation, together with the fact that $S\left(\mathbb{R}^{3}\right)$ is dense in $H^{\mu, s}\left(\mathbb{R}^{3}\right)$ for any pair of $\mu$ and $s$, ensures that the extension of $\left.\widetilde{\Gamma}_{0}(z)\right|_{S\left(\mathbb{R}^{3}\right)}$ to $H^{\mu, s}\left(\mathbb{R}^{3}\right)$ is independent of $\mu$ and $s$ in a certain sense. However, we shall not discuss about the uniqueness of the extension any longer. In the discussions below, we shall mostly deal with the extension of $\left.\widetilde{\Gamma}_{0}(z)\right|_{S\left(\mathbb{R}^{3}\right)}$ to $H^{-1, s}\left(\mathbb{R}^{3}\right)$.

Lemma 4.4 Let $s$, $s^{\prime}$ satisfy the same assumption as in Lemma 4.2, and let $\mu \in \mathbb{R}$. Then $\widetilde{\Gamma}_{0}(z)$ is a $B\left(\mu-2, s ; \mu,-s^{\prime}\right)$-valued continuous function on $\Pi_{(0,+\infty)}$. 
Proof. We first note that

$$
\langle D\rangle^{2} \Gamma_{0}(z) u=u+(z+1) \Gamma_{0}(z) u
$$

for $u \in S\left(\mathbb{R}^{3}\right)$ and $z \in \mathbb{C} \backslash[0,+\infty)$; cf. Jensen and Kato [18, Lemma 2.1]. (See (1.4) for the definition of $\langle D\rangle$.) We then combine (4.26) with Lemma 4.3 , and obtain the conclusion if we appeal to the fact that $S\left(\mathbb{R}^{3}\right)$ is dense in $H^{\mu-2, s}\left(\mathbb{R}^{3}\right)$.

What we shall need in the rest of the paper is a variant of Lemma 4.4, namely a version for four-component vector-valued functions, with $\mu=1$ in the form described in Proposition 4.1 below. Thus $\widetilde{\Gamma}_{0}(z)$ in Proposition 4.1 denotes a copy of $\widetilde{\Gamma}_{0}(z)$; see (1.5).

Proposition 4.1 Let $s, s^{\prime}$ satisfy the same assumption as in Lemma 4.2. Then $\widetilde{\Gamma}_{0}(z)$ is a $\mathcal{B}\left(-1, s ; 1,-s^{\prime}\right)$-valued continuous function on $\Pi_{(0,+\infty)}$.

\subsection{The resolvent of the free Dirac operator $\boldsymbol{H}_{0}$}

In view of (4.4), it is convenient for us to introduce the following operator valued-functions $\Omega_{0}^{+}(z)$ defined on $\overline{\mathbb{C}}_{+}$and $\Omega_{0}^{-}(z)$ on $\overline{\mathbb{C}}_{-}$as follows:

$$
\Omega_{0}^{ \pm}(z)=\widetilde{\Gamma}_{0}\left(z^{2}\right), \quad z \in \overline{\mathbb{C}}_{ \pm},
$$

in other words,

$$
\Omega_{0}^{ \pm}(z)= \begin{cases}\Gamma_{0}\left(z^{2}\right) & \text { if } z \in \mathbb{C}_{ \pm} \\ \Gamma_{0}^{ \pm}\left(\lambda^{2}\right) & \text { if } z=\lambda \geq 0 \\ \Gamma_{0}^{\mp}\left(\lambda^{2}\right) & \text { if } z=\lambda \leq 0\end{cases}
$$

It follows from Proposition 4.1 that $\Omega_{0}^{+}(z)\left(\right.$ resp. $\left.\Omega_{0}^{-}(z)\right)$ is a $\mathcal{B}\left(-1, s ; 1,-s^{\prime}\right)$ valued continuous function on $\overline{\mathbb{C}}_{+}\left(\right.$resp. $\left.\overline{\mathbb{C}}_{-}\right)$. Also, it follows from (4.20) that

$$
\Omega_{0}^{+}(0)=\Omega_{0}^{-}(0)=\widetilde{\Gamma}_{0}(0) .
$$

In order to get expressions of the extended resolvents of the free Dirac operator $H_{0}$ in terms of $\Omega_{0}^{ \pm}(z)$ introduced in (4.28), we shall exploit the decomposition (4.4) and a boundedness estimate of $H_{0}$ in some weighted Sobolev spaces which is given as follow.

Lemma 4.5 Let $\mu$ and $s^{\prime}$ be in $\mathbb{R}$. Then

$$
H_{0} \in \mathcal{B}\left(\mu,-s^{\prime} ; \mu-1,-s^{\prime}\right) \text {. }
$$


Proof. To prove the lemma, it is sufficient to show that

$$
\langle x\rangle^{-s^{\prime}}\langle D\rangle^{\mu-1} D_{j}\langle D\rangle^{-\mu}\langle x\rangle^{s^{\prime}}=\langle x\rangle^{-s^{\prime}} D_{j}\langle D\rangle^{-1}\langle x\rangle^{s^{\prime}}
$$

$(j=1,2,3)$ is a bounded operator from $L^{2}\left(\mathbb{R}^{3}\right)$ to $L^{2}\left(\mathbb{R}^{3}\right)$. This fact is a direct consequence of Umeda [33, Lemma 2.1].

Lemma 4.6 Let $s, s^{\prime}>1 / 2$, and $s+s^{\prime}>2$. Then $R_{0}(z) \in \mathcal{B}\left(-1, s ; 0,-s^{\prime}\right)$ is continuous in $z \in \mathbb{C}_{ \pm}$. Moreover, as $\mathcal{B}\left(-1, s ; 0,-s^{\prime}\right)$-valued functions, they can possess continuous extensions $R_{0}^{ \pm}(z)$ to $\overline{\mathbb{C}}_{ \pm}$respectively, and

$$
R_{0}^{ \pm}(z)=\left(H_{0}+z\right) \Omega_{0}^{ \pm}(z), \quad z \in \overline{\mathbb{C}}_{ \pm} .
$$

Proof. We shall give the proof only for $z \in \overline{\mathbb{C}}_{+}$. The proof for $z \in \overline{\mathbb{C}}_{-}$is similar.

As was mentioned before Lemma $4.5, \Omega_{0}^{+}(z)$ is a $\mathcal{B}\left(-1, s ; 1,-s^{\prime}\right)$-valued continuous function on $\overline{\mathbb{C}}_{+}$. Combining this fact with (4.4), (4.5), the definition (4.27) (or (4.28)) of $\Omega_{0}^{+}(z)$, Proposition 4.1 and Lemma 4.5 with $\mu=1$, we see that $R_{0}(z)=\left(H_{0}+z\right) \Omega_{0}^{+}(z) \in \mathcal{B}\left(-1, s ; 0,-s^{\prime}\right)$ for any $z \in$ $\mathbb{C}_{+}$. Now it is evident that the second assertion of the lemma follows from Proposition 4.1 and Lemma 4.5 with $\mu=1$.

Combining (4.30) with (4.29), we obtain a corollary to Lemma 4.6.

Corollary 4.1 Under the same assumption and the same notation as in Lemma 4.6,

$$
R_{0}^{+}(0)=R_{0}^{-}(0)=H_{0} \widetilde{\Gamma}(0) \quad \text { in } \mathcal{B}\left(-1, s ; 0-s^{\prime}\right) .
$$

Remark 4.2 In [17], Iftimovici and Măntoiu showed that the limiting absorption principle for the the free Dirac operator $H_{0}=\alpha \cdot D+m \beta, m>$ 0 , in $\mathcal{B}(0,1 ; 0,-1)$ holds on the whole real line. With the result exhibited in Lemma 4.6, together with the result in [17], the limiting absorption principle for the the free Dirac operator $H_{0}=\alpha \cdot D+m \beta$ has been established for all $m \geq 0$.

Lemma 4.7 For $f \in \mathcal{S}$ and $z \in \mathbb{C}_{ \pm}$

$$
\begin{aligned}
& R_{0}(z) f(x) \\
& =\int_{\mathbb{R}^{3}}\left(i \frac{\alpha \cdot(x-y)}{|x-y|^{2}} \pm z \frac{\alpha \cdot(x-y)}{|x-y|}+z I_{4}\right) \frac{e^{ \pm i z|x-y|}}{4 \pi|x-y|} f(y) d y .
\end{aligned}
$$


Proof. We first recall (4.25), which we can write as

$$
\Gamma_{0}\left(z^{2}\right) f(x)=\int_{\mathbb{R}^{3}} \frac{e^{ \pm i z|y|}}{4 \pi|y|} f(x-y) d y, \quad f \in \mathcal{S}, z \in \mathbb{C}_{ \pm} .
$$

We then combine (4.27) and (4.30), and make differentiation under the integral sign in (4.32), which gives

$$
R_{0}(z) f(x)=\int_{\mathbb{R}^{3}} \frac{e^{ \pm i z|y|}}{4 \pi|y|}\left(\alpha \cdot D_{x}+z I_{4}\right) f(x-y) d y, \quad z \in \mathbb{C}_{ \pm}
$$

Noting the fact that

$$
D_{x} f(x-y)=-D_{y}(f(x-y)),
$$

and making integration by parts on the right hand of (4.33) implies that

$$
\begin{array}{r}
R_{0}(z) f(x)=\int_{\mathbb{R}^{3}}\left(i \frac{\alpha \cdot y}{|y|^{2}} \pm z \frac{\alpha \cdot y}{|y|}+z I_{4}\right) \frac{e^{ \pm i z|y|}}{4 \pi|y|} f(x-y) d y \\
\left(z \in \mathbb{C}_{ \pm}\right) .
\end{array}
$$

A change of variables in (4.34) yields (4.31). (See also Thaller [32, p. 39].)

Proposition 4.2 For $f \in \mathcal{S}$

$$
R_{0}^{+}(0) f=R_{0}^{-}(0) f=A f,
$$

where $A$ is the singular integral operator defined by (3.1).

Proof. In view of Corollary 4.1, we only need to give the proof for $R_{0}^{+}(0)$.

Let $f \in \mathcal{S}$, and let $\left\{z_{n}\right\} \subset \mathbb{C}_{+}$be a sequence such that $z_{n} \rightarrow 0$ as $n \rightarrow \infty$. It follows from Lemma 4.6 that $R_{0}\left(z_{n}\right) f \rightarrow R_{0}^{+}(0) f$ in $\mathcal{L}^{2,-s^{\prime}}$ as $n \rightarrow \infty$. This fact implies that there exists a subsequence $\left\{z_{n^{\prime}}\right\} \subset\left\{z_{n}\right\}$ such that

$$
R_{0}\left(z_{n^{\prime}}\right) f(x) \rightarrow R_{0}^{+}(0) f(x) \text { a.e. } x \in \mathbb{R}^{3} \text { as } n^{\prime} \rightarrow \infty .
$$

On the other hand, Lemma 4.7, together with Lebesgue's convergence theorem, implies that

$$
R_{0}\left(z_{n}\right) f(x) \rightarrow \int_{\mathbb{R}^{3}} i \frac{\alpha \cdot(x-y)}{4 \pi|x-y|^{3}} f(y) d y=A f(x) \quad \text { as } n \rightarrow \infty
$$


for each $x \in \mathbb{R}^{3}$. The conclusion of the proposition now follows from (4.35) and (4.36).

Lemma 4.8 Let $s, s^{\prime}>1 / 2$, and $s+s^{\prime}>2$. Then $A$ can be continuously extended to an operator in $\mathcal{B}\left(-1, s ; 0,-s^{\prime}\right)$.

Proof. Since $\mathcal{S}$ is dense in $\mathcal{H}^{-1, s}$, Lemma 4.6 and Proposition 4.2 directly imply the lemma.

In the rest of the paper, we shall denote the extension in Lemma 4.8 by $A$ again. Thus we have

$$
R_{0}^{+}(0)=R_{0}^{-}(0)=A \quad \text { in } \mathcal{B}\left(-1, s ; 0,-s^{\prime}\right) .
$$

Proposition 4.3 Let $s>1 / 2$. Then

$$
H_{0} A g=g
$$

for all $g \in \mathcal{L}^{2, s}$.

Proof. Let $g \in \mathcal{L}^{2, s}$ be given. We then start with the fact that

$$
\left(H_{0}-z\right) R_{0}(z) g=g \quad\left(\forall z \in \mathbb{C}_{+}\right) .
$$

Choose $s^{\prime}>1 / 2$ so that $s+s^{\prime}>2$. We see from Lemmas 4.6, 4.8 and Proposition 4.2 that

$$
R_{0}\left(\frac{i}{n}\right) g \rightarrow R_{0}^{+}(0) g=A g \quad \text { in } \mathcal{L}^{2,-s^{\prime}} \quad \text { as } n \rightarrow \infty .
$$

Lemma 4.5, with $\mu=0$, and (4.39) imply that

$$
\left(H_{0}-\frac{i}{n}\right) R_{0}\left(\frac{i}{n}\right) g \rightarrow H_{0} A g \quad \text { in } \mathcal{H}^{-1,-s^{\prime}} \quad \text { as } n \rightarrow \infty .
$$

Since, by (4.38),

$$
\left(H_{0}-\frac{i}{n}\right) R_{0}\left(\frac{i}{n}\right) g=g \quad \text { for } \forall n,
$$

we find that (4.40) yields (4.37).

We shall need Lemma 2.4 of Jensen and Kato [18], which we shall rewrite in a suitable form to our setting (cf. Lemma 4.9 below), where the operators $-\Delta$ and $\widetilde{\Gamma}_{0}(0)$ act on four-component vector functions. The reader should note that $\widetilde{\Gamma}_{0}(0)$ is the same as $G_{0}$ in Jensen-Kato's paper. See (4.19) and (4.20). 
Lemma 4.9 (Jensen-Kato) Let $s>1 / 2$. Then

(i) $(-\Delta) \widetilde{\Gamma}_{0}(0) g=g$ for all $g \in \mathcal{H}^{-1, s}$.

(ii) $\widetilde{\Gamma}_{0}(0)(-\Delta) f=f$ if $f \in \mathcal{L}^{2,-3 / 2}$ and $(-\Delta) f \in \mathcal{H}^{-1, s}$.

Proposition 4.4 Let $s>1 / 2$. Then $\widetilde{\Gamma}_{0}(0) H_{0} g=$ Ag for all $g \in \mathcal{L}^{2, s}$.

Proof. Let $g \in \mathcal{L}^{2, s}$ be given. Noting that $H_{0}^{2}=-\Delta$ (cf. (4.6)), we have

$$
(-\Delta) A g=H_{0}\left(H_{0} A g\right)=H_{0} g
$$

where we have used Proposition 4.3 in the second equality. Since $H_{0} g \in$ $\mathcal{H}^{-1, s}$ by Lemma 4.5 , it follows from (4.41) that $(-\Delta) A g \in \mathcal{H}^{-1, s}$.

On the other hand, we find, by Lemma 4.8, that $A g \in \mathcal{L}^{2,-3 / 2}$, because we can choose $s^{\prime}$ so that $1 / 2<s^{\prime} \leq 3 / 2$ and $s+s^{\prime}>2$. (Choose $s^{\prime}$ so that $\max (s, 2-s)<s^{\prime} \leq 3 / 2$. $)$

Now we can apply Lemma 4.9 (ii) with $f$ replaced by $A g$, and obtain

$$
\widetilde{\Gamma}_{0}(0)(-\Delta) A g=A g \text {. }
$$

It follows from (4.41) that the left hand side of (4.42) equals $\widetilde{\Gamma}_{0}(0) H_{0} g$. This proves the conclusion of the proposition.

Proof of Theorem 4.1. Put

$$
g=H_{0} f .
$$

By assumption of the theorem, we see that $g \in \mathcal{L}^{2, s}$ for some $s>1 / 2$. It follows from Proposition 4.4 that $A g=\widetilde{\Gamma}_{0}(0) H_{0} g$, i.e.,

$$
A H_{0} f=\widetilde{\Gamma}_{0}(0) H_{0} H_{0} f=\widetilde{\Gamma}_{0}(0)(-\Delta) f .
$$

Since $(-\Delta) f=H_{0} g \in \mathcal{H}^{-1, s}$ by Lemma 4.5 , it follows from assertion (ii) of Lemma 4.9 that $\widetilde{\Gamma}_{0}(0)(-\Delta) f=f$. Thus $A H_{0} f=f$.

\section{Proof of the main theorems}

Proof of Theorem 2.1. We first prove assertion (i). Let $f$ be a zero mode of the operator $H$ in (1.1). Then we have

$$
H f=(\alpha \cdot D+Q(x)) f=0, \quad f \in \operatorname{Dom}(H)=\mathcal{H}^{1} .
$$

It follows from (5.1) and Assumption (A) that

$$
H_{0} f=(\alpha \cdot D) f=-Q(x) f \in \mathcal{L}^{2, \rho} .
$$


(Recall (4.1) for the definition of $H_{0}$.) Since $\rho>1>1 / 2$ by assumption of the theorem, we can apply Theorem 4.1 to $(5.2)$ and get

$$
f=A H_{0} f=-A Q f .
$$

It follows from (5.3) and Lemma 3.1 that $f \in \mathcal{L}^{2} \cap \mathcal{L}^{6}$. It follows from (5.3) again and Lemma 3.3 that $f \in \mathcal{L}^{\infty}$. This fact, together with (5.3) and Assumption (A), implies that

$$
\begin{aligned}
|f(x)| & \leq \frac{3}{4 \pi} \int_{\mathbb{R}^{3}} \frac{1}{|x-y|^{2}}|Q(y) f(y)| d y \\
& \leq C\|f\|_{\mathcal{L}^{\infty}} \int_{\mathbb{R}^{3}} \frac{1}{|x-y|^{2}\langle y\rangle^{\rho}} d y .
\end{aligned}
$$

Noting that $\rho>1$ by assumption, and applying the inequalities in (4.8) to the integral in (5.5), we get

$$
|f(x)| \leq C\|f\|_{\mathcal{L}^{\infty}} \begin{cases}\langle x\rangle^{-\rho+1} & \text { if } 1<\rho<3 \\ \langle x\rangle^{-2} \log (1+\langle x\rangle) & \text { if } \rho=3 \\ \langle x\rangle^{-2} & \text { if } \rho>3 .\end{cases}
$$

If $\rho>3$, we have already obtained the desired estimate. If $1<\rho \leq 3$, we plug the inequalities in (5.6) into (5.4). We thus get

$$
|f(x)| \leq C\|f\|_{\mathcal{L}^{\infty}} \int_{\mathbb{R}^{3}} \frac{1}{|x-y|^{2}\langle y\rangle^{2 \rho-1}} d y
$$

if $1<\rho<3$, and

$$
|f(x)| \leq C\|f\|_{\mathcal{L}^{\infty}} \int_{\mathbb{R}^{3}} \frac{\log (1+\langle y\rangle)}{|x-y|^{2}\langle y\rangle^{\rho+2}} d y
$$

if $\rho=3$. We find that the inequalities in (4.8) applied to the integrals in (5.7) and (5.8) yields

$$
|f(x)| \leq C\|f\|_{\mathcal{L}^{\infty}} \begin{cases}\langle x\rangle^{-2(\rho-1)} & \text { if } 1<\rho<2, \\ \langle x\rangle^{-2} \log (1+\langle x\rangle) & \text { if } \rho=2, \\ \langle x\rangle^{-2} & \text { if } 2<\rho \leq 3 .\end{cases}
$$

Hence, if $2<\rho \leq 3$, we have shown the desired estimate. If $1<\rho \leq 2$, we repeat the same argument again, actually as many times as we wish. 
Summing up, we can obtain the estimate

$$
|f(x)| \leq C_{N}\|f\|_{\mathcal{L}^{\infty}} \begin{cases}\langle x\rangle^{-N(\rho-1)} & \text { if } 1<\rho<1+2 / N \\ \langle x\rangle^{-2} \log (1+\langle x\rangle) & \text { if } \rho=1+2 / N \\ \langle x\rangle^{-2} & \text { if } 1+2 / N<\rho\end{cases}
$$

for any positive integer $N$, where $C_{N}$ is a constant depending on $N$. It is straightforward that for a given $\rho>1$ in Assumption (A), we can choose $N$ so that $1+(2 / N)<\rho$. This fact, together with (5.10), implies assertion (i).

We next prove assertion (ii) by utilizing (5.3):

$$
f(x)=-\int_{\mathbb{R}^{3}} i \frac{\alpha \cdot(x-y)}{4 \pi|x-y|^{3}} Q(y) f(y) d y .
$$

Let $x_{0}$ be any point in $\mathbb{R}^{3}$, and let $\varepsilon>0$ be given. We choose $r>0$ so that

$$
\int_{|y| \leq 2 r} \frac{1}{|y|^{2}} d y<\varepsilon
$$

We then decompose $f(x)$ into two parts:

$$
\begin{aligned}
f(x) & =-\left(\int_{B(x, 2 r)}+\int_{E(x, 2 r)}\right) i \frac{\alpha \cdot(x-y)}{4 \pi|x-y|^{3}} Q(y) f(y) d y \\
& =: f_{b}(x)+f_{e}(x),
\end{aligned}
$$

where

$$
B(x, 2 r)=\{y|| x-y \mid \leq 2 r\}, \quad E(x, 2 r)=\{y|| x-y \mid>2 r\} .
$$

Since each $\alpha_{j}$ is a unitary matrix, it follows from (5.11) and (5.12) that

$$
\left|f_{b}(x)\right|<\frac{3}{4 \pi} C_{q} C_{f} \varepsilon \quad \text { for } \forall x \in \mathbb{R}^{3},
$$

where $C_{q}$ is a constant determined by (1.6) in Assumption (A) and $C_{f}$ is a constant described in the inequality (2.1), which we have just proved in the first half of the proof. It follows from the definition of $f_{e}(x)$ that

$$
\begin{aligned}
& f_{e}(x)-f_{e}\left(x_{0}\right) \\
& =\int_{\mathbb{R}^{3}}\left\{1_{E\left(x_{0}, 2 r\right)}(y) \frac{\alpha \cdot\left(x_{0}-y\right)}{4 \pi\left|x_{0}-y\right|^{3}}-1_{E(x, 2 r)}(y) \frac{\alpha \cdot(x-y)}{4 \pi|x-y|^{3}}\right\} \\
& \times Q(y) f(y) d y .
\end{aligned}
$$


To apply Lebesgue's convergence theorem to the integral in (5.14), we need the following two facts that

$$
\left|x_{0}-y\right| \geq r \quad \text { if }\left|x_{0}-x\right|<r,|x-y|>2 r
$$

and that

$$
|x-y| \geq \frac{2}{3}\left|x_{0}-y\right| \quad \text { if }\left|x_{0}-x\right|<r,|x-y|>2 r
$$

(use the inequality $|x-y| \geq\left|x_{0}-y\right|-\left|x_{0}-x\right|$ ). We can deduce from (5.15) and (5.16) that

$$
\begin{aligned}
& \left|1_{E(x, 2 r)}(y) \frac{\alpha \cdot(x-y)}{4 \pi|x-y|^{3}} Q(y) f(y)\right| \\
& \leq 1_{E\left(x_{0}, r\right)}(y) \frac{3}{4 \pi}\left(\frac{2}{3}\left|x_{0}-y\right|\right)^{-2}|Q(y) f(y)|
\end{aligned}
$$

whenever $\left|x_{0}-x\right|<r$. It is straightforward that the estimate (5.17) implies

the integrand in (5.14)|

$$
\leq 1_{E\left(x_{0}, r\right)}(y) \frac{3}{4 \pi}\left(1+\left(\frac{3}{2}\right)^{2}\right)\left|x_{0}-y\right|^{-2}|Q(y) f(y)|
$$

whenever $\left|x_{0}-x\right|<r$. In view of (1.6) in Assumption (A) and the inequality (2.1), the function on the right hand side of (5.18) is integrable on $\mathbb{R}^{3}$. Thus, we can apply Lebesgue's convergence theorem to the integral in (5.14), and conclude that

$$
\lim _{x \rightarrow x_{0}}\left(f_{e}(x)-f_{e}\left(x_{0}\right)\right)=0 .
$$

Combining (5.19) with both (5.12) and (5.13) yields

$$
\limsup _{x \rightarrow x_{0}}\left|f(x)-f\left(x_{0}\right)\right| \leq 2 \times \frac{3}{4 \pi} C_{q} C_{f} \varepsilon .
$$

Since $\varepsilon$ was arbitrary, this completes the proof of assertion (ii).

Proof of Theorem 2.2. Let $f$ satisfy the assumption of the theorem: $f \in$ $\mathcal{L}^{2,-s}$ for some $s$ with $0<s \leq \min \{3 / 2, \rho-1\}$. In the same manner as in (5.2) and (5.3), we can show that

$$
H_{0} f=(\alpha \cdot D) f=-Q f \in \mathcal{L}^{2, \rho-s},
$$


and that

$$
f=-A Q f .
$$

Note that $s \leq 3 / 2$ and $\rho-s \geq 1>1 / 2$, which we have used to apply Theorem 4.1 in showing (5.21). Since $Q f \in \mathcal{L}^{2, \rho-s}, \rho-s \geq 1$, we see from (5.21) and Lemma 3.2 that $f \in \mathcal{L}^{2}$. This fact, together with (5.20) and Lemma 4.1, gives the conclusion of the theorem.

Acknowledgment T.U. would like to express his gratitude to Michael Loss for the hospitality during his visit to Georgia Institute of Technology, USA, in April, 2002. Discussions with Michael were one of the motivations of the present paper. Also, he would like to express his thanks to the Department of Mathematics, the University of Alabama at Birmingham, USA, for their hospitality. Part of the present paper was done during his stay there in March and September, 2006. Finally the authors appreciate invaluable comments by Michael Loss, Kenji Yajima and the referee. Kenji's comments helped us improve the main theorems of the previous version of the present paper.

\section{References}

[1] Adam C., Muratori B. and Nash C., Zero modes of the Dirac operator in three dimensions. Phys. Rev. D 60 (1999), 125001-1-125001-8.

[2] Adam C., Muratori B. and Nash C., Degeneracy of zero modes of the Dirac operator in three dimensions. Phys. Lett. B 485 (2000), 314-318

[ 3 ] Adam C., Muratori B. and Nash C., Multiple zero modes of the Dirac operator in three dimensions. Phys. Rev. D 62 (2000), 085026-1-085026-9.

[4] Aharonov Y. and Casher A., Ground state of a spin-1/2 charged particle in a twodimensional magnetic field. Phys. Rev. A 19 (1979), 2461-2462.

[5] Balinsky A.A. and Evans W.D., On the zero modes of Pauli operators. J. Funct. Analysis 179 (2001), 120-135.

[6] Balinsky A.A. and Evans W.D., On the zero modes of Weyl-Dirac operators and their multiplicity. Bull. London Math. Soc. 34 (2002), 236-242.

[7] Balinsky A.A. and Evans W.D., Zero modes of Pauli and Weyl-Dirac operators. Advances in differential equations and mathematical physics (Birmingham, AL, 2002), 1-9, Contemp. Math. 327, Amer. Math. Soc., Providence, RI. 2003.

[ 8 ] Balslev E. and Helffer B., Limiting absorption principle and resonances for the Dirac operator. Adv. Appl. Math. 13 (1992), 186-215.

[9] Bugliaro L., Fefferman C. and Graf G.M., A Lieb-Thirring bound for a magnetic Pauli Hamiltonian, II. Rev. Mat. Iberoamericana 15 (1999), 593-619.

[10] Eckardt K.-J., Scattering theory for Dirac operators. Math. Z. 139 (1974), 105-131. 
[11] Elton D.M., The local structure of zero mode producing magnetic potentials. Commun. Math. Phys. 229 (2002), 121-139.

[12] Erdös L. and Solovej J.P., The kernel of Dirac operators on $\mathbb{S}^{3}$ and $\mathbb{R}^{3}$. Rev. Math. Phys. 13 (2001), 1247-1280.

[13] Erdös L. and Solovej J.P., Uniform Lieb-Thirring inequality for the threedimensional Pauli operator with a strong non-homogeneous magnetic field. Ann. Henri Poincaré 5 (2004), 671-741.

[14] Erdös L. and Solovej J.P., Magnetic Lieb-Thirring inequalities with optimal dependence on the field strength. J. Statist. Phys. 116 (2004), 475-506.

[15] Erdös L. and Vougalter V., Pauli operator and Aharonov-Casher theorem for measure valued magnetic fields. Commun. Math. Phys. 225 (2002), 399-421.

[16] Fröhlich J., Lieb E.H. and Loss M., Stability of Coulomb systems with magnetic fields. I. The one-electron Atom. Commun. Math. Phys. 104 (1986), 251-270.

[17] Iftimovici A. and Măntoiu M., Limiting absorption principle at critical values for the Dirac operator. Lett. Math. Phys. 49 (1999), 235-243.

[18] Jensen A. and Kato T., Spectral properties of Schrödinger operators and time-decay of the wave functions. Duke Math. J. 46 (1979), 583-611.

[19] Kalf H. and Yamada O., Essential self-adjointness of n-dimensional Dirac operators with a variable mass term. J. Math. Phys. 42 (2001), 2667-2676.

[20] Kalf H., Okaji T. and Yamada O., Absence of eigenvalues of Dirac operators with potentials diverging at infinity. Math. Nachr. 259 (2003), 19-41.

[21] Kuroda S.T., An introduction to scattering theory. Lecture Note Series, vol. 51, Aarhus University, Aarhus, 1980.

[22] Kuroda S.T., Spectral theory II. Iwanami Shoten, Tokyo, 1979.

[23] Loss M. and Yau H.T., Stability of Coulomb systems with magnetic fields. III. Zero energy bound states of the Pauli operators. Commun. Math. Phys. 104 (1986), 283290.

[24] Persson M., On the Dirac and Pauli operators with several Aharonov-Bohm solenoids. Lett. Math. Phys. 78 (2006), 139-156.

[25] Pladdy C., Asymptotics of the resolvent of the Dirac operator with a scalar shortrange potential. Analysis 21 (2001), 79-97.

[26] Pladdy C., Saitō Y. and Umeda T., Resolvent estimates of the Dirac operators. Analysis 15 (1995), 123-149.

[27] Pladdy C., Saitō Y. and Umeda T., Radiation condition for Dirac operators. J. Math. Kyoto Univ. 37 (1997), 567-584.

[28] Reed M. and Simon B., Methods of modern mathematical physics II; Fourier analysis, self-adjointness. Academic Press, New York, 1975.

[29] Rozenblum G. and Shirokov N., Infiniteness of zero modes for the Pauli operator with singular magnetic field. J. Funct. Analysis 233 (2006), 135-172.

[30] Schmidt K.M. and Yamada O., Spherically symmetric Dirac operators with variable mass and potentials infinity at infinity. Publ. Res. Inst. Math. Sci. Kyoto Univ. 34 (1998), 211-227. 
[31] Stein E.M., Singular integrals and differential properties of functions. Princeton University Press, Princeton, New Jersey, 1970.

[32] Thaller B., The Dirac equation. Springer-Verlag, Berlin Heidelberg, 1992.

[33] Umeda T., The action of $\sqrt{-\Delta}$ on weighted Sobolev spaces. Lett. Math. Phys. 54 (2000), 301-313.

[34] Umeda T., Generalized eigenfunctions of relativistic Schrödinger operators I. Electron. J. Diff. Eqns. (127) 2006 (2006), 1-46.

[35] Yamada O., On the spectrum of Dirac operators with unbounded potential at infinity. Hokkaido Math. J. 26 (1997), 439-449.

Y. Saitō

Department of Mathematics

University of Alabama at Birmingham

Birmingham, AL 35294, USA

E-mail: saito@math.uab.edu

T. Umeda

Department of Mathematical Sciences University of Hyogo

Shosha, Himeji 671-2201, Japan

E-mail: umeda@sci.u-hyogo.ac.jp 